\title{
Research Progress in Ordered Nanomaterials via Magnetic Field Induced Preparation
}

\author{
Yunhao Liu ${ }^{1,2}$, Mingzhe $\mathrm{Lv}^{1,2}$, Chao Wang ${ }^{1}$, Zuyu He${ }^{1}$, Chuang Zhou ${ }^{1}$, Ziming Yang ${ }^{1}$, Puwang Li $^{1 \mathrm{a}}$ \\ ${ }^{1}$ South Subtropical Crops Research Institute, Chinese Academy of Tropical Agricultural Sciences, Zhanjiang, Guangdong 524091, China \\ ${ }^{2}$ Agricultural Products Processing Research Institute, Chinese Academy of Tropical Agricultural Sciences, Zhanjiang, Guangdong 524001, \\ China
}

\begin{abstract}
Ordered nanomaterials are widely concerned for their excellent performance in mechanics, electricity, optics and magnetism. Magnetic field induced self-assembly is widely used for the preparation of ordered nanomaterials, which has the advantages of indirect contact with the reaction system, controllable adjustment of magnetic field. By using this preparation method, it can realize the alignment of nanomaterials without affecting the comprehensive performance of each component in the system. In this paper, we reviewed magnetic field induced self-assembly of metal nanomaterials, oxide nanomaterials and nanocomposites. We also looked forward to the future research direction.
\end{abstract}

\section{Introduction}

The unique physical and chemical properties of nanomaterials make it possible to develop materials with various special functions ${ }^{[1]}$. With the development of preparation technology, people can prepare a variety of nanomaterials, but for most of the preparation of nanomaterials, it is still difficult to overcome the problem of easy agglomeration. The agglomeration of nanomaterials results in disordered arrangement of structures, which limits the further practical application performance of nanomaterials. Ordered structure materials are formed by directional alignment or self-assembly according to certain rules in the process of preparation. At present, the preparation of ordered materials is mainly realized by self-assembly technology. Surfactants or templates are usually used to induce self-assembly in the process of material synthesis to form ordered structure materials. The self-assembly can also be induced by using an external field, that is, under the action of force ${ }^{[2]}$, electricity ${ }^{[3]}$, magnetism ${ }^{[4]}$ and other external fields, the nanoparticles can complete self-assembly to form new ordered structure nanomaterials.

As an external force, the magnetic field can induce the directional alignment and self-assembly of materials in a non-contact condition, forming an ordered structure ${ }^{[5]}$. For magnetic nanomaterials, the use of external magnetic field to control the self-assembly of the material has significant advantages such as good controllability, reusability, and freedom from physical or chemical interactions ${ }^{[6,7]}$. In this paper, we reviewed the progress in the preparation of ordered structure nanomaterials by using external magnetic fields.

\section{Metal nanomaterials}

Magnetic metal nanomaterial is a kind of important magnetic nanomaterials. Through physical and chemical methods, magnetic metal nanomaterials with different sizes and morphologies can be easily prepared. However, their size and morphology will also affect their properties. In order to obtain ideal properties, a lot of researchers have taken advantage of the action of external magnetic field to induce the synthesis of magnetic metal nanomaterials.

Cheng et al. ${ }^{[8]}$ prepared Co nanoparticles in colloidal solution induced by magnetic field (0.05T). Co Nanoparticles with a diameter of $15 \mathrm{~nm}$ were assembled into linear nanochains along the direction of the external magnetic field; after removing the magnetic field, the chain structure was still maintained, but lost linearity, and folded into a spiral three-dimensional structure under slight agitation. Sun et al. ${ }^{[9]}$ successfully prepared fibrous magnetic metal Ni by liquid-phase reduction method with ethylene glycol as solvent under the action of external magnetic field. The results showed that the product $\mathrm{Ni}$ was chain fiber without magnetic field; when the external uniform magnetic field was $0.3 \mathrm{~T}$, the product $\mathrm{Ni}$ became linear fiber, and the magnetic nanoparticles $\mathrm{Ni}$ in the process of external magnetic field-induced reaction were arranged in the direction of the magnetic field and grew continuously along the direction.

Krajewski et al. ${ }^{[10]}$ studied a new technology of magnetic field-induced synthesis of magnetic Fe-Co alloy $\left(\mathrm{Fe}_{0.75} \mathrm{Co}_{0.25}, \quad \mathrm{Fe}_{0.50} \mathrm{Co}_{0.50}\right.$ and $\left.\mathrm{Fe}_{0.25} \mathrm{Co}_{0.75}\right)$ linear nanochains (Figure 1), using $\mathrm{NaBH}_{4}$ aqueous solution as reducing agent, through a simple one-step magnetic fieldinduced chemical cooperative reduction reaction of three different concentrations of $\mathrm{Fe}^{2+}$ and $\mathrm{Co}^{2+}$ ion precursor 
solutions, to prepare the amorphous bimetallic Fe Co linear nanostructures with ideal Fe/CO ratio which have special bimetallic alloy core and very thin oxide shell structure. The Fe-Co alloy material is a ferromagnetic material, and its magnetic properties are related to its structural properties and chemical composition. As the cobalt content increases, its saturation magnetization decreases significantly.

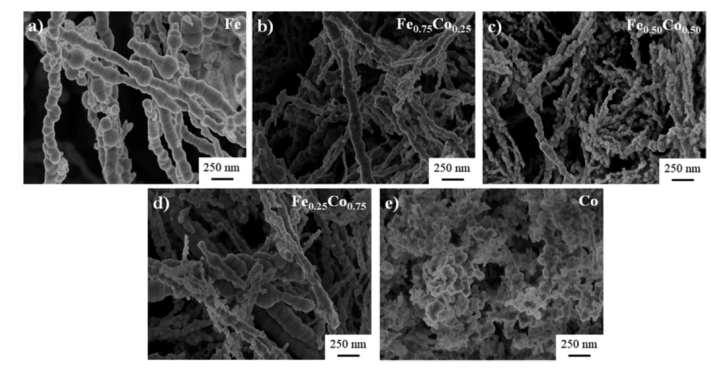

Fig1. SEM images of (a) $\mathrm{Fe}$, (b) $\mathrm{Fe}_{0.75} \mathrm{Co}_{0.25}$, (c) $\mathrm{Fe}_{0.50} \mathrm{Co}_{0.50}$, (d) $\mathrm{Fe}_{0.25} \mathrm{Co}_{0.75}$ wire-like nanostructures, and (e) Co nanoparticles [10]

\section{Oxide nanomaterials}

Li et al. ${ }^{[11]}$ used one-step thermal decomposition route to rapidly synthesize octahedral magnetite $\left(\mathrm{Fe}_{3} \mathrm{O}_{4}\right)$ nanocrystals. Through adjusting the ratio of precursor to surfactant, the average particle size can be well controlled from $8 \mathrm{~nm}$ to $430 \mathrm{~nm}$. Under the action of an external weak magnetic field $(0.06 \mathrm{~T})$ in the horizontal and vertical directions, through a simple solvent evaporation selfassembly process, he acquired a two-dimensional monolayer or three-dimensional micro-superstructure consisting of nanocrystals of $21 \mathrm{~nm}$ in size, all of which had a high degree of crystal orientation.

Zhu et al. ${ }^{[12]}$ used the co-precipitation method to prepare wheat ear-like $\mathrm{Fe} 3 \mathrm{O} 4$ under the action of an external magnetic field in chitosan solution. The ordered structure was formed by ordered alignment and selfassembly of spindle shaped particles with length of $0.5 \sim 1.5$ $\mathrm{m}$ and diameter of 100-500nm, which were composed of $\mathrm{Fe}_{3} \mathrm{O}_{4}$ nanowires with a length of $100 \sim 500 \mathrm{~nm}$ and a diameter of $5 \sim 10 \mathrm{~nm}$ arranged in parallel. The results showed that under the action of external magnetic field, the $\mathrm{Fe}_{3} \mathrm{O}_{4}$ crystal nucleus in the solution continuously grew along the direction of magnetic field, and then form nanowires. Due to the characteristics of aspect ratio, the nanowires continue to self-assemble to form spindle-like "wheat grain" basic structural unit, and further aligned and self-assembled into multi-level structure $\mathrm{Fe}_{3} \mathrm{O}_{4}$ material with wheat spike shape under the action of external magnetic field.

\section{Nanocomposites}

Nanocomposites are kind of multi-phase materials which have special properties and can be artificially designed. Through adjusting the composition and ratio of the composite materials, we can achieve the complementary advantages of different materials and obtain the outstanding comprehensive performance. At the same time, it also solved the problems of single magnetic nanoparticle self-assembly such as poor functionality and difficult structure fixation ${ }^{[13]}$. Taking nanoparticles as the structural unit, the ordered nanocomposite materials are compounded with other matrix materials, thus forming nanocomposites in ordered arrangement. Since they have ordered structure, the acquired composites also ensure the properties of nano fillers. Compared with the disordered composites, their properties are greatly improved; the commonly applied preparation method is that firstly modify the magnetic nanoparticles or the surface of the materials, and then under the effect of the external magnetic field, induce the nanomaterials' directional alignment in the matrix, thus preparing nanocomposites with ordered structure to improve all the performances of composite materials ${ }^{[14]}$.

\subsection{Inorganic nanocomposites}

Jia et al. ${ }^{[15]}$ used magnetic field-induced self-assembly to prepare $\mathrm{Fe}_{3} \mathrm{O}_{4}$ microspheres with $\mathrm{SiO}_{2}$ bonded to anisotropic structure. First, $\mathrm{Fe}_{3} \mathrm{O}_{4}$ microspheres were synthesized by a modified reduction reaction between $\mathrm{FeCl}_{3}$ and ethylene glycol in a solvothermal system, and then tetraethyl silicate (TEOS) was hydrolyzed and polycondensed in a mixture of isopropanol and hydration ammonia, with $\mathrm{Fe}_{3} \mathrm{O}_{4}$ microspheres being coated with $\mathrm{SiO}_{2}$. Then $\mathrm{Fe}_{3} \mathrm{O}_{4}-\mathrm{SiO}_{2}$ microspheres were obtained by wet precipitation-induced self-assembly by applying $1 \mathrm{~T}$ external magnetic field vertically above the $\mathrm{Fe}_{3} \mathrm{O}_{4}-\mathrm{SiO}_{2}$ colloidal solution. The scanning electron microscopy showed that microspheres with diameters in the range of $200-550 \mathrm{~nm}$ self-assembled along the direction of the external magnetic field to form a "corn-cob"-like quasione-dimensional structure.

There are many studies and reports on the synthesis strategy of magnetic nanoparticles self-assembly under a magnetic field for preparing kinds of inorganic nanocomposite materials, such as $\mathrm{SiO}_{2}$ coated $\mathrm{Fe}_{3} \mathrm{O}_{4}-\mathrm{Ag}$ nanowire magnetic film ${ }^{[16]}$, core-shell structured chaintyped $\mathrm{Fe}_{3} \mathrm{O}_{4} / \mathrm{C} /$ Red $\mathrm{P}$ nanocomposites ${ }^{[17]}$. These materials all have well characteristics of alignment self-assembly and adjustable magnetic properties. This kind of green preparation technology utilizing an external magnetic field to induce the preparation of ordered structural materials has controllability and excellent reaction efficiency, which can broaden the precise composition of complex material structures. 


\subsection{Polymer nanocomposites}

Wu et al. ${ }^{[18]}$ utilized a simple and effective co-precipitation method in a relatively weak magnetic field to attach magnetic nanoparticles $\left(\mathrm{Fe}_{3} \mathrm{O}_{4}\right)$ to carbon nanofibers (CNFs), forming a new magnetic-carbon nanofiber hybrid $\left(\mathrm{Fe}_{3} \mathrm{O}_{4} @ \mathrm{CNFs}\right.$, to make the carbon nanofibers aligned into a chain structure in the epoxy resin. Wen et al. [19] firstly prepared composite nanoparticles of magnetic $\mathrm{Fe}_{3} \mathrm{O}_{4}$ and polyaniline $\left(\mathrm{Fe}_{3} \mathrm{O}_{4} @ \mathrm{PANI}\right)$, and then inducing the self-assembly of the nanoparticles through the use of magnetic field. The nanoparticles showed ordered alignment self-assembly on the surface of the magnetic glassy carbon electrode (MGCE), forming a highly sensitive uniform molecularly imprinted polymer (MIPs) film.

\subsection{Carbon nanotube composites}

Kimura et al. ${ }^{[20]}$ put forwarded a method of preparing anisotropic carbon nanotubes (MWNTs) polymer composites through the induction of a magnetic field. By polymerizing under a $10 \mathrm{~T}$ magnetic field, MWNTs are arranged in the monomer solution, and then carry out the chemical polymerization of monomer matrix, thus realizing the alignment in the nanomaterials polymer matrix. The performance tests of magnetic properties, electrical conductivity and elastic modulus all showed obvious anisotropy, and demonstrated that the orientation of MWNTs in the polymer matrix is parallel to the magnetic field under the induction of a magnetic field.

Garmestani et al. ${ }^{[21]}$ studied the alignment of carbon nanotubes in polymers. The presence of a magnetic field during the resin solidification process made the molecules to be oriented along the direction of the applied field. When the magnetic field strength was 25T, the degree of alignment was the highest, showing effective alignment with the direction of the magnetic field. The carbon nanotube epoxy resin composite material also produced a similar phenomenon under the action of a $25 \mathrm{~T}$ magnetic field, arranged along the direction of the magnetic field, but it was rougher than the fracture surface of a single epoxy resin. Studies showed that in addition to the magnetic anisotropy of carbon nanotubes, the high orientation of epoxy resin is conducive to the alignment of carbon nanotubes along the direction of the magnetic field.

\subsection{Graphene nanocomposites}

As the key components of graphene based composites, oriented graphene composites have huge application potential in many fields. In view of the unique thermal conductivity, graphene-oriented composite materials are equipped with excellent thermal conductivity and anisotropy, and the thermal conductivity of anisotropy in parallel and vertical directions have obvious differences. However, because the magnetic susceptibility of the polymer matrix is relatively low, a higher magnetic field is usually required to achieve the alignment of its graphene microplates (GNPs) ${ }^{[22]}$. Under the action of a magnetic field, the magnetic filler can easily conduct alignment in the polymer matrix. As a result, to modify the magnetic function of GNPs is necessary. Magnetic modification can significantly increase the magnetic susceptibility of GNPs ${ }^{[23]}$. Usually, it is to combine GNPs with magnetic particles, and $\mathrm{Fe}_{3} \mathrm{O}_{4}$ nanoparticles are the most common magnetic functional particles.

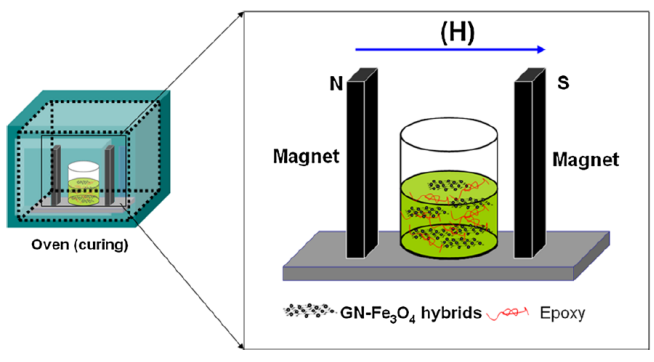

Fig2. .Schematic for the synthesis of epoxy/GNS- $\mathrm{Fe}_{3} \mathrm{O}_{4}$ composites by magnetic alignment ${ }^{[25]}$

Liu et al. ${ }^{[24]}$ synthesized $\mathrm{Fe}_{3} \mathrm{O}_{4}$ nanorods coated and reduced GNPs hybrid particles $\left(\mathrm{GNP}-\mathrm{Fe}_{3} \mathrm{O}_{4}\right)$ through a simple co-precipitation method, and then under the action of a weak magnetic field, making the $\mathrm{GNP}-\mathrm{Fe}_{3} \mathrm{O}_{4}$ hybrid particles aligned to prepare bismaleimide composite material. $\mathrm{Fe}_{3} \mathrm{O}_{4}$ nanoparticles attached to graphene can effectively reduce the agglomeration of $\mathrm{Fe}_{3} \mathrm{O}_{4}$ nanoparticles and the re-stacking of GNPs. Once acting in the magnetic field (Figure 2), the magnetic moment imposed on the $\mathrm{Fe}_{3} \mathrm{O}_{4}$ nanoparticles forces the GNP- $\mathrm{Fe}_{3} \mathrm{O}_{4}$ hybrid particles to be aligned along the direction of the magnetic field ${ }^{[25]}$. Jiao et al. ${ }^{[26]}$ applied wet chemical coprecipitation method to coat magnetic $\mathrm{Fe}_{3} \mathrm{O}_{4}$ nanoparticles on the surface of nanographene microplates to achieve graphene's magnetic modification. It showed that magnetic graphene microplates (m-GNPs) could be arranged alone, and could also be embedded in a polymer matrix under a weak magnetic field $(40 \mathrm{mT})$ to prepare highly ordered m-GNPs/EP composites.

\section{Prospect}

At present, the application of the magnetic field-induced self-assembly technology still needs in-depth research. Currently, a set of perfect magnetic field-induced selfassembly theory system that can be used to guide the construction of ordered nanomaterials is lacked to acquire and develop nanomaterials more in line with people's design concept. More research should focus on ordered nanocomposites, of which the important feature is that the size is in the nanoscale range and has specific properties of nanomaterials. However, the aggregation of 
nanoparticles themselves cannot be ignored. How to conduct the ideal design and controllable preparation of the size, structure and morphology of nanoparticles, and overcome the self-aggregation of nanoparticles to improve the stability of the composite system, have always been major challenges in the research of nanocomposites. With the continuous development of nanotechnology, researchers' continuous in-depth research on the magnetic field-induced preparation technology of complex and multifunctional composite nanomaterials, and the research on low-cost, rapid preparation, equipment simplification, have further expanded the research value in more fields. Magnetic field-induced preparation technology will surely become a simple and universal preparation method for ordered nanomaterials and will have broader application fields.

\section{Acknowledgments}

This work was supported by the Central Public-inter est Scientific Institution Basal Research Fund for Ch inese Academy of Tropical Agricultural Sciences (No. 1630122020003, No.1630122017009, No.16301220170 $11)$.

\section{References}

1. Vigderman L, Khanal B P, Zubarev E R. Adv Mater, 2012, 24(36): 4811 4841.

2. 2 Chang M Y, Wang W H, Chung Y C. J Mater Chem, 2011, 21(13): 4966 4970.

3. Liu M, Obi O, Lou J, Chen Y J, Cai Z H, Stoute S, Espanol M, Lew M, Situ X D, Ziemer K S, Harris V G, Sun N X. Adv Funct Mater, 2009, 19(11): $1826 \sim 1831$.

4. Lu Y, Dong L, Zhang L C, Su Y D, Yu S H. Nano Today, 2012, 7(4): 297 315.

5. Wang M S, Yin Y D. J Am Chem Soc, 2016, 138(20): 6315 6323.

6. Pileni M P, Ngo A T. Chem Phys Chem, 2005, 6(6): 1027 1034.

7. Erb R M, Libanori R, Rothfuchs N, Studart A R. Science, 2012, 335(6065): 199 204.

8. Cheng G J, Romero D R, Fraser G T, Hight Walker A R. Langmuir, 2005, 21(26):12055 12059.

9. Sun W S, Li H, Liu Y, Zhao X C, Cheng J W, Wen S L. J Magn Mater Devices, 2015(2): 10 13. (in Chinese)

10. Krajewski M, Liou S C, Chiou W A, Tokarczyk M, Małolepszy A, Płocińska M, Witecka M, Lewińska S, Ślawska-Waniewska A. Cryst Growth Des, 2020, 20 (5):3208 3216.

11. Li L, Yang $\mathrm{Y}$, Ding J, Xue J M. Chem Mater, 2010, 22 (10): 3183 3191.

12. Zhu S Q, Wang Y, Wu S Y, Xiong X P, Xiamen Univ Nat Sci, 2016, 55(02): 174 177. (in Chinese)

13. Zhou X L.NanChang Hangkong University, 2015.(in
Chinese)

14. Niu Y. Harbin Institute of Technology, 2014.(in Chinese)

15. Jia B P, Gao L. Scripta Mater, 2007, 56(8): 677 680.

16. Hassan M, Zhan H J , Wang J L, Liu J W, Chen J F. ChemistryOpen, 2020, 9(5): 588 592.

17. Qin G H, Duan J Y, Yang Y C, Liu F S. ACS Appl Mater Interfaces, 2018, 10(7): 6441 6452.

18. . Wu S Y, Ladani R B, Zhang J, Kinloch A J, Zhao Z H, Ma J, Zhang X H, Mouritz A P, Ghorbani K, Wang C H. Polymer, 2015, 68:25 34.

19. Wen T T, Zhu W Y, Xue C, Wu J H, Han Q, Wang X, Zhou X M, Jiang H J. Biosens Bioelectron, 2014, 56:180 185.

20. Kimura T, Ago H, Tobita M, Ohshima S, Kyotani M, Yumura M. Adv Mater, 2002, 14(19):1380 1383.

21. Garmestani H, Al-Haik M S, Dahmen K, Tannenbaum R, Li D, Sablin S S, Hussaini M Y. Adv Mater, 2003, 15(22):1918 1921.

22. Zhang Z X, Qu J Y, Feng Y Y, Feng W. Compos Commu, 2018, 9:33 41.

23. Thévenot J, Oliveira H, Sandre O, Lecommandoux S. Chem Soc Rev, 2013, 42(17):7099-7116.

24. Liu C, Yan H X, Chen Z Y, Yuan L X, Liu T Y. J Mater Chem A, 2015, 3 (19): 10559-10565.

25. Yan H Y, Tang Y X, Long W, Li Y F. J Mater Sci, 2014 , 49: 5256-5264.

26. Jiao W C, Shioya M, Wang R G, Yang F, Hao L F, Niu Y, Liu W B, Zheng L, Yuan F, Wan L, He X D. Compos Sci Technol, 2014, 99:124 130. 